\title{
CRISPER-RNA Guided Gene Editing and Implications in Endogenous Genes Activation
}

\author{
Maria Qamar ${ }^{2}$, Kinza Tanvir ${ }^{1}$, Sadia Akbar ${ }^{1}$, Usman Ghani ${ }^{*}$, Haider Ali ${ }^{2}$, Muhammad Bilal ${ }^{2}$, Asim Rehman ${ }^{2}$, Zulaikha \\ Arif $^{1}$, Sumaira Batool ${ }^{1}$
}

${ }^{1}$ Department of Biochemistry, University of Agriculture, Faisalabad, Pakistan

${ }^{2}$ Department of Biochemistry, Government College University, Faisalabad, Pakistan

\author{
DOI: $\underline{10.36348 / \mathrm{sijb} .2020 . \mathrm{v} 03 \mathrm{i} 03.006}$ \\ | Received: 16.03.2020 | Accepted: 24.03.2020 | Published: 30.03 .2020 \\ *Corresponding author: Usman Ghani
}

\section{Abstract}

Short guide RNAs of catalytically inactive CRISPR associated 9 nuclease uses to express endogenous genes in human cells and bacteria. Chimeric nucleases help in precise and efficient genetic modifications leads to double strand breakage which can be repaired by DNA repair mechanism by homology directed repair and non-homology end joining. Here, we describe that single or multiple gRNAs direct dCas9 fused to a VP64 transcriptional activation domain to enhance the expression of endogenous human genes. Light-activated CRISPR-Cas9 effector (LACE) system induces dynamic regulation of genes in the presence of blue light. Targeted activation of endogenous neurogenic genes in transgenic mice leads to direct and efficient conversion of astrocytes into functional neurons in vivo. CRISPR-on can activate the endogenous ILIRN, SOX2, and OCT4 genes. Activation of genes was achieved by clusters of 3-4 sgRNAs that bind to the proximal promoters, result in their synergistic action in gene induction. Moreover, it fused with transcriptional activators in endogenous human INS (silenced gene with a fully methylated promoter). Multiplexed gene editing helps in metabolic engineering in yeast and $\mathrm{CHO}$ mammalian host. In this review we describe the applications and development of Cas9 for a variety of research or translational applications.

Keywords: CRISPR-Cas9, IL1RN, SOX2, sgRNA, dCas9-VP64, CRISPRi, GFP, LACE.

Copyright @ 2020: This is an open-access article distributed under the terms of the Creative Commons Attribution license which permits unrestricted use, distribution, and reproduction in any medium for non-commercial use (NonCommercial, or CC-BY-NC) provided the original author and source are credited.

\section{INTRODUCTION}

The chronicles for the discovery of CRISPR starts in 1987.The presence of a 29 nucleotide repeat in Escherichia coli, which were interrupted by unrelated, non-repetitive short sequences (spacers). CRISPR/Cas9 is emerging a most favorite arsenal owing to its easy adaptability, versatility, and cost effectiveness. The CRISPR-Cas system is an adaptive immune system which provides resistance against foreign genetic elements in prokaryotes [1]. The effector arm of type II CRISPR/Cas systems relies on a single protein, called Cas9, that is guided to specific DNA targets by two small RNA molecules called the trans activating CRISPRRNA (tracrRNA) and CRISPR RNA (crRNA) [2].

One potential application of the dCas 9 protein would be to specifically activate individual human genes by fusing dCas 9 to a potent transactivation domain, such as that found on VP64. Cas9 also has the potential to be used as a vehicle to deliver functional modules to specific sites on the genome [3].
The CRISPR array consists of several repeat sequences, interspaced by spacers. These spacers are unique segments obtained from foreign DNA which provide sequence specific immunity against foreign DNA elements. A cluster of Cas genes are generally located next to such repeat-spacer units. New spacers can also be introduced into the CRISPR locus during infection so that it can act as a memory during a subsequent encounter with the same invaders [4]. The length of repeat sequences can vary among different loci of the same genome. Recent findings reveal that the repeat sequences range from 18-50nucleotides (nt) whereas spacer sequences range from 17-84 nt long [5].

Cas 1 and Cas 2 are universal in all CRISPR loci, whereas Cas3, Cas9 and Cas10 are specific for type I, II and III CRISPR-Cas systems respectively. Among the CRISPR-Cas types, the type II system has received more attention than the rest because of its ability to induce double strand breaks in the target DNA. This modified version of CRISPR Cas9 system 
employs Cas proteins guided by gRNA to cleave the target DNA sequence. There are two different components in the CRISPR/ Cas9 system: gRNA and an endonuclease (Cas9). The CRISPR activity requires a CRISPR locus including an array of repeat-spacer sequences and a set of CRISPR associated genes (Cas genes) which code for proteins essential for processing, functioning and cleavage activity. The entire defence process can be classified into three phases as described below.

The acquisition phase of CRISPR constructs the genetic memory of the cell. In this phase, new spacers obtained from the invading plasmids or foreign DNAs during the first encounter are incorporated into the array of CRISPR which allows the cell to adapt against the invaders present in the environment. This is divided into two steps, the protospacer selection and the generation of spacer. It is followed by incorporation into the array and synthesis of a new repeat sequence. Cas1 and Cas 2 are the two most important proteins in spacer acquisition phase of CRISPR. They work as a complex, where a single dimer of Cas2 binds to two dimers of Cas 1 to perform the activity [4].
The CRISPR transcription initiates in the leader region which contains the promoter elements, binding sites for regulatory proteins and elements important for spacer integration. A primary transcript, pre-crRNA is generated from the CRISPR array which is further processed into smaller units corresponding to a single [1].

Once the crRNAs are generated, they recognize the invading target sequences through base complementarity. Following this, the crRNAs along with Cas proteins perform the target degradation process. The foreign nucleic acid is cleaved by crRNA and Cas proteins at sites complementary to the crRNA spacer sequence. Figure-1 showing defence mechanism of CRISPR Cas system in bacteria.

In the immediate future, the use of the CRISPR-Cas9 system will revolutionize military medical sciences and advance the fundamental knowledge of anti-pathogen defense, radiation protection, tissue homeostasis. Cas9/sgRNA mediated genome editing provides new therapeutic strategies for infectious diseases, wound healing and tissue regeneration.

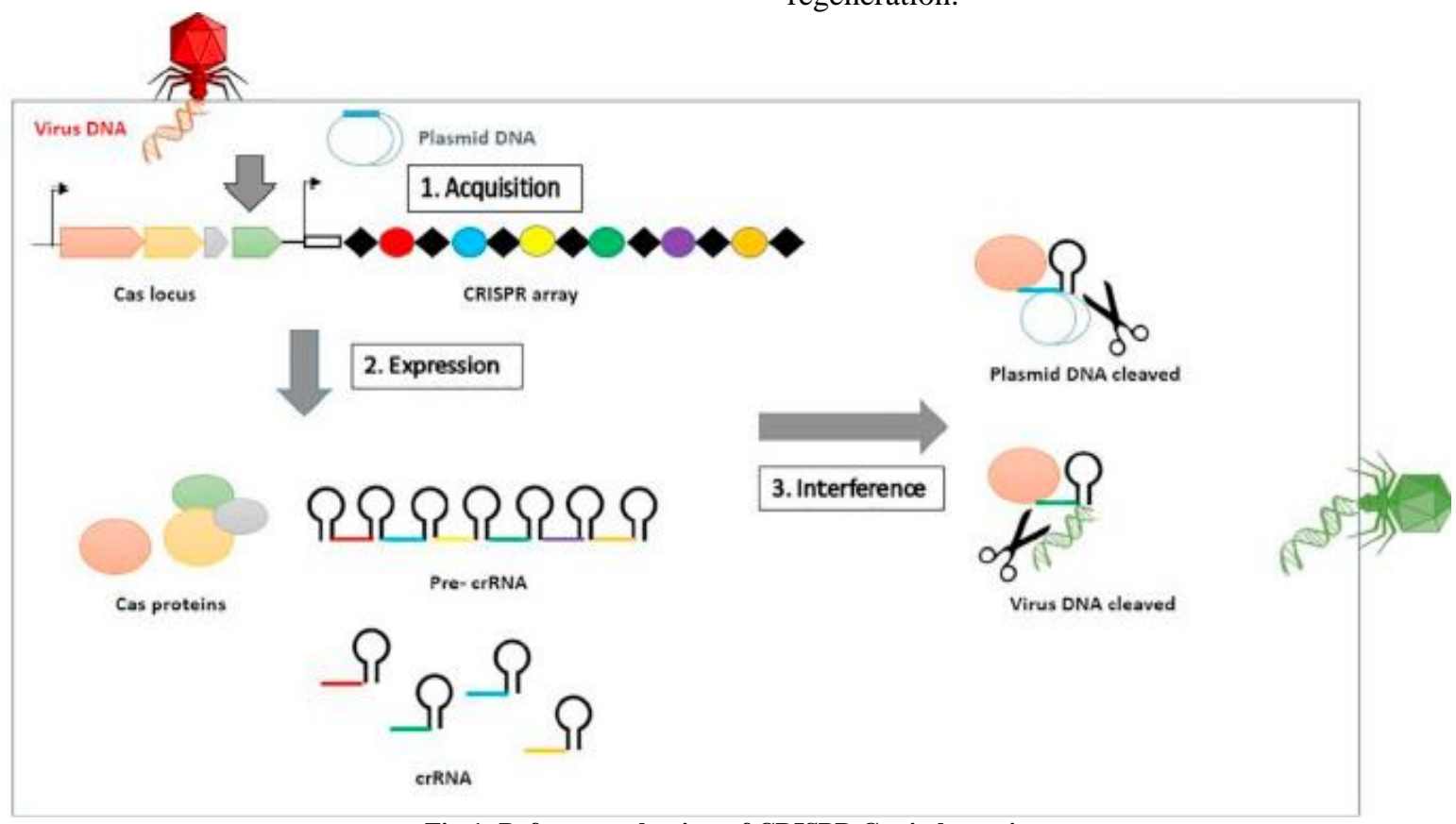

Fig-1: Defence mechanism of CRISPR Cas in bacteria

\section{Endogenous Human Genes Activation}

The Streptococcus pyogenes Cas9 can be directed by a 100-nucleotide (nt) single-guide RNA (sgRNA) to a target genomic DNA sequence that is complementary to the first $20 \mathrm{nt}$ of the sgRNA [6]. RNA-mediated recruitment of a catalytically inactive form of Cas9 (here referred to as dCas9) can induce repression of endogenous genes in bacterial and human cells [7]. Fusions of dCas9 to effector domains have been used to activate reporter genes in Escherichia coli and human cells and to repress endogenous genes in human cells [8].
dCas9-VP64 fusion protein consisting of the synthetic VP64 activation domain11 linked to the C terminus of dCas 9 and tested the capacity of this fusion to activate expression of the human VEGFA gene. We targeted the dCas9-VP64 protein to specific sites in the genome of human embryonic kidney 293 (HEK293) cells using 16 revalidated sgRNAs that we had observed could be expressed in HEK293 cells and could induce Cas9-mediated cleavage at their target sites. These sgRNAs bind sequences within three DNase I hypersensitive sites located upstream, downstream or at the VEGFA gene transcription start 
site. Out of these 16 sgRNAs (named V1-V16), 15 induced significant increases in VEGFA protein expression when co-expressed with dCas9-VP64 in HEK293 cells [9].

\section{Targeted genome editing in algae}

One major problem in genetic improvement of micro algal systems is due to the dismal frequency/lack of homologous recombination in nuclear genome [10]. Engineered nucleases include Zinc finger nucleases (ZFNs), transcription activator-like effector nucleases (TALENs), mega nucleases, and and clustered regularly interspaced short palindromic sequences (CRISPR)/ CRISPR-associated protein 9 for precise cuts in the genome at loci of choice [11]. This CRISPR arrays allow the bacteria to "remember'" the intruder's genetic material and displays the adaptive nature of this immune system [12].

The first CRISPR based genome editing was reported in $\mathrm{C}$ reinhardtii, the chassis for majority of physiological, evolutionary and molecular biology studies in algae. To ensure proper expression of the Cas9 nuclease, Cas9 from Streptococcus pyogenes was codon optimised and put under control of (CaMV) 35S promoter and the nopaline synthetase gene (nos) termination region [13]. Mutations in the FKB12 or peptidyl-prolyl cis-trans isomerase gene results in tolerance to the antibiotic Rapamycin. In the same expression cassette crRNA and tracrRNA were fused together to form a chimeric sgRNA, which was driven by the RNA polymerase III dependant U6 promoter. Four genes were targeted of three were exogenous, namely mutant GFP mutant hygromycin resistance gene and the mutant Gluc gene and the endogenous FKB12 gene [14]

\section{CRISPRi mediated gene repression}

The ability of CRISPRi was then tested on the endogenous gene Phosphoenolpyruvate carboxylase, responsible for the formation of oxaloacetate (OAA) from phosphoenolpyruvate (PEP), an important substrate for tricarboxylic acid cycle [15]. Previous studies have found that during lipid accumulation, CrPEPC2 RNA levels were down-regulated and the carbon flux was diverted to lipid accumulation in RNAi mutants of CrPEPC2 [16]. The CRISPRi CrPEPC2 mutant produced $28.5 \%$ dry cell weight (DCW) lipid along with a productivity of $34.9 \mathrm{mg} / \mathrm{L} /$ day, translating into an increase of $74.4 \%$ and $28.5 \%$ in lipid and productivity, respectively than the wild type [17].

\section{Multiplexed activation of endogenous genes}

Transcription factors regulate gene expression by binding to specific DNA sequences at the enhancer and promoter regions of target genes, and modulate transcription through their effector domains [18]. Artificial transcription factors (ATFs) have been generated by fusing various functional domains to a DNA binding domain engineered to bind to the genes of interest, thereby modulating their expression [19]. A subsequent study demonstrated a more efficient gene repression in eukaryotes by dCas 9 fused with a transcription repression domain or exogenous transgene activation when fused with an activation domain [20].

The major advantage of this system is that only one dCas 9 activator is required to activate multiple genes individually or simultaneously and that its DNA binding specificity is determined by sgRNAs, which are designed based on simple RNA/DNA complementarity. Efficient activation of endogenous genes could be achieved by three to five sgRNAs binding within 300 bp region upstream of TSS. We achieved robust endogenous gene activation using the stronger activation domain VP160. CRISPR-on can activate the endogenous ILIRN, SOX2, and OCT4 genes. The most efficient gene activation was achieved by clusters of 3-4 sgRNAs binding to the proximal promoters, suggesting their synergistic action in gene induction. Significantly, when sgRNAs targeting multiple genes were simultaneously introduced into cells, robust multiplexed endogenous gene activation was achieved. Genomewide expression profiling demonstrated high specificity of the system [21].

\section{Endogenous human INS gene activation}

The CRISPR-on system fused with transcriptional activators (dCas9-VP160) activated endogenous human INS, which is a silenced gene with a fully methylated promoter. Similarly, synergistic effect on gene activation when multiple single guide RNAs were used, and the transcriptional activation was maintained [22].

CRISPR-on system allowed for the activation of endogenous genes, we co-transfected NIH3T3 mouse cells with an expression plasmid consisting of dCas9 fused to 10 copies of VP16 (dCas9-VP160) and three sgRNAs targeting the endogenous Oct4 transcription factor (T1, T2 and NT3, previously tested . CRISPR-on system in NIH3T3 was able to turn on Oct4 expression. These results confirmed that the system could be used to activate endogenous genes [23].

\section{Light-inducible CRISPR-Cas9 system for control of endogenous gene activation \\ Genome engineering technologies have} enabled activation or repression of endogenous genes in mammalian cells using synthetic transcription factors that can be targeted to almost any DNA sequence. Fusion of one heterodimer zing protein to a ZFP18 or TALE4 and fusion of its binding partner to a transcriptional activation domain, such as VP64, enables light-dependent recruitment of the activation domain to the DNA sequence targeted by the ZFP or TALE but this process is too much laborious. we fused the light-inducible heterodimer zing proteins CRY2 and CIB1 from Arabidopsis thaliana to the VP64 transactivation domain and either the $\mathrm{N}$ - or $\mathrm{C}$ - terminus 
of dCas9, the catalytically inactive form of Cas9 (D10A, H840A) [3].

\section{Light-activated CRISPR-Cas9 effector} (LACE) system that induces transcription of endogenous genes in the presence of blue light. This was accomplished by fusing the light-inducible heterodimer zing proteins CRY2 and CIB1 to a transactivation domain and the catalytically inactive dCas9, respectively. The versatile LACE system can be easily directed to new DNA sequences for the dynamic regulation of endogenous genes. The combination of CRY2FL-VP64 with dCas9-CIBN or CIBN-dCas9 showed detectable levels (10- to 100-fold greater than those generated by dCas9 fusions to a single CIBN domain) of gene activation in the light [24].

The CRISPR-Cas9 technology, an efficient, inexpensive, fast-to-design, and easy-to-use genomic editing tool, hasbeen rapidly applied in many fields, ranging from basic biology to translational medicine [25].

\section{CRISPR-Cas9 in the generation of animal models}

Many recent studies have shown that CRISPRCas9 technology could be used for rapidly generating targeted genome modifications in the germ lines of various model organisms [26]. Microinjection of Cas9encoding mRNA and customizable sgRNA into onecell stage zebra fish embryos is able to efficiently modify the target genes in vivo in a simple, rapid and scalable manner [27]. Co-injection of Cas 9 mRNA and sgRNAs targeting different genes into mouse zygotes generates mutant mice with biallelic mutations, confirming that CRISPR/Cas-mediated gene editing could be used for the simultaneous disruption of multiple genes with high efficiency [27]. Multiplexed activation of endogenous genes can be achieved by injecting a two-component transcriptional activator including a nuclease-dead Cas9 protein fused with a transcriptional activation domain and sgRNA stargeting gene promoters [21]. CRISPR-Cas9 technology has been used for efficient genome engineering in many other model organisms, including Drosophila ,Caenorhabditis elegans, Axolotl, Xenopus tropicalis , rat and pig [25].

\section{CRISPR-Cas9 role in functional genomic screening}

Functional genomic screening is largely used for identifying the essential genes for a specific cellular process. A genome-scale CRISPR-Cas9 knockout (GeCKO) library has been developed and successfully used for screening genes essential for cell viability in cancer and pluripotent stem cells and for genes associated with the resistance to vemurafenib, a drug for late-stage melanoma [28]. A CRISPR-Cas-based knockout library has been applied to identify the host genes mediating the cellular responses to anthrax and diphtheria toxins [25].
A CRISPRi system consisting of a catalytically inactive Cas9 and a guide RNA has been shown to specifically and efficiently repress the transcription of target genes in Escherichia coli and mammalian cells [29] whereas a catalytically inactive Cas9 fused with a transcriptional activation domain has been used to activate the expression of specific endogenous genes [7].

A very recent study has shown that CRISPRCas9 complexes with synergistic activation mediators are able to achieve robust, single sgRNAmediated gene up regulation at endogenous genomic loci. When used with an sgRNA library, the engineered Cas9 activation complexes can activate multiple genes simultaneously, up regulate long intragenic non-coding RNA transcripts and identify genes conferring resistance to a BRAF inhibitor through a genome-wide dCas9-based transcription activation screening in a melanoma model [30].

In vivo simultaneous activation of multiple endogenous genes in the brain of transgenic mice using CRISPR-dCas9- activator

Targeting a single gene is usually insufficient to model the pathological processes. Thus, SPH-mousebased in vivo multiplex activation potentially provides a platform for better disease modeling [31]. We generated a transgenic mouse using an improved dCas9 system that enables simultaneous and precise in vivo transcriptional activation of multiple genes and long noncoding RNAs in the nervous system. As proof of concept, we were able to use targeted activation of endogenous neurogenic genes in these transgenic mice to directly and efficiently convert astrocytes into functional neurons in vivo. Using the piggyback (PB) transposon system, we generated a SPH transgenic mouse containing HA-tagged dCas9 fused with10xGCN4, which is linked with p65-HSF1 and EGFP in tandem via P2A and T2A, respectively [32].

After transfection with Cre-expression plasmids or infection with Cre-expression virus, dCas9 and EGFP expression could be effectively and specifically induced in vitro and in vivo.We first targeted two genes encoding neuronal transcription factors, Ascll and Neurog2, in primary astrocytes. Both Ascll and Neurog2 were significantly unregulated with differents gRNA combinations. Using SPH mice, we achieved robust activation of multiple genes in the liver with a mixture of sgRNAs. CRISPR-dCas9 activators have been developed for multiple transcriptional activations [33].

For genes with limited activation efficiency, modest activations of some genes, such as Mecp2and Shank3, could also be sufficient to induce physiological changes of neurons31-33. It is also important to note that we examined the efficacy of SPH system in neurons and astrocytes, which are the representative 
cell types in the brain. Collectively, these results highlight the advantages and potential of using SPH mice to modulate complex genetic networks in the intact brain [34].

\section{Specific induction of endogenous viral restriction factors using CRISPR/Cas-derived transcriptional activators}

Mutants of the RNA-guided DNA binding protein Cas9 that have lost their DNA cleavage activity could be used to recruit transcription activation domains to specific promoters. Recently, the recruitment of multiple transcription activation domains by a single sgRNA, modified to contain MS2-derived stem loops that recruit fusion proteins consisting of the MS2 coat protein linked to transcription activation domains, was reported to induce otherwise silent cellular genes [35].

Synergistic activation mediators" can induce the expression of two restriction factors, APOBEC3G (A3G) and APOBEC3B (A3B), in human cells that normally lack these proteins. $A 3 G$ is susceptible to degradation by the HIV-1 Vif protein, whereas A3B is resistant to Vif. As a result, only the induced A3B inhibited wild-type HIV-1 infectivity. However, both induced factors blocked the replication of a Vifdeficient HIV-1 mutant [36]. SAM consisting of not only a dCas/VP64 fusion but also a modified sgRNA scaffold containing two MS2 coat protein binding sites, each of which could bind two copies of a fusion protein consisting of MS2 coat protein linked to two transcription activation domains derived from the NF$\kappa \mathrm{B}$ p65 subunit and HSF1. SAMs were reported to efficiently activate cellular genes when guided to a single binding site located $<200 \mathrm{bp} 5^{\prime}$ to the transcription start site. The use of a single sgRNA per promoter would clearly facilitate gain-of-function screens for cellular genes that can regulate virus replication. Wild-type viruses have often evolved mechanisms to overcome inhibition by specific restriction factors, including inhibition of $\mathrm{A} 3 \mathrm{G}$ by HIV$1 \mathrm{Vif}$ and of tethering by HIV-1 Vpu. Moreover, many restriction factors are not constitutively expressed but are instead induced upon viral infection, including numerous IFN-stimulated genes (ISGs) [37].

The recruitment of SAMs to either the Apo lipoprotein B mRNA-editing enzyme catalytic polypeptide (APOBEC) APOBEC3B (A3B) or APOBEC 3G(A3G) promoter in human cells that normally do not express either restriction factor results in a dramatic and specific induction in either A3B or A3G expression [38].

\section{CRISPR-Cas9 in correction of genetic disorders}

A very recent study has shown that the CRISPR-Cas9 system can be used to modify an EGFP transgene or the endogenous Crygc gene in spermatogonial stem cells (SSCs). The modified SSCs carrying a corrected Crygc mutation can undergo spermatogenesis and produce offspring with the corrected phenotype at an efficiency of 100\% [39]. Interestingly, a similar strategy using the CRISPR-Cas9 technology has successfully corrected the cystic fibrosis transmembrane conductor receptor (CFTR) locus by homologous recombination in cultured intestinal stem cells of cystic fibrosis human patients, demonstrating that primary adult stem cells derived from patients with a single-gene hereditary defect could be corrected by CRISPR/Cas9 mediated homologous recombination, suggesting a promising strategy for gene therapy in human patients [40].

\section{CRISPR-Cas9 in the treatment of infectious diseases}

Recently, studies have shown that the CRISPR-Cas9 system can eliminate the HIV-1 genome and prevent new HIV infection [23]. When transfected into HIV-1 provirus-integrated human cells, an sgRNA expression vector targeting the long terminal repeats (LTR) of HIV- 1 efficiently cleaves and mutates LTR target sites and suppresses LTR-driven viral gene expression. In addition, this system has been shown to delete viral genes from the host cell chromosome [41] Cas9/sgRNAs efficiently inactivate HIV gene expression and replication in latently infected cells, including microglial, promonocytic and $\mathrm{T}$ cells. Significantly, Cas9/sgRNA mediated genome editing has been shown to immunize cells to prevent HIV-1 infection [23].

Recent advances in genome engineering technologies based on the CRISPR-associated RNA guided endonuclease Cas9 are enabling the systematic interrogation of mammalian genome function. Using this system, DNA sequences within the endogenous genome and their functional outputs are now easily edited or modulated in virtually any organism of choice [42].

\section{Future Development of Cas9-Based Genome Engineering Technologies \\ Transcription control by cas 9 dCas 9 binding} alone to DNA elements may repress transcription by steric ally hindering RNA polymerase machinery [29]. Likely by stalling transcriptional elongation. This CRISPR-based interference, or CRISPRi, works efficiently in prokaryotic genomes but is less effective in eukaryotic cells. The repressive function of CRISPRI can be enhanced by tethering dCas 9 to transcriptional repressor domains such as KRAB or SID effectors, which promote epigenetic silencing. Although the requirement for multiple $\mathrm{sgRNAs}$ to achieve efficient transcription activation is potentially advantageous for increased specificity, screening applications employing libraries of sgRNAs will require highly efficient and specific transcriptional control using individual guide RNAs [20]. 


\section{Cas9 as a Therapeutic Molecule for Treating Genetic Disorders}

For dominant- negative disorders in which the affected gene is haplo sufficient (such as transthyretinrelated hereditary amyloidosis or dominant forms of retinitis pigment sum), it may also be possible to use NHEJ to inactivate the mutated allele to achieve therapeutic benefit. For allele-specific targeting, one could design guide RNAs capable of distinguishing between single nucleotide polymorphism (SNP) variations in the target gene, such as when the SNP falls within the PAM sequence [20]. Recently, hydrodynamic delivery of plasmid DNA encoding Cas9 and sgRNA along with a repair template into the liver of an adult mouse model of tyrosinemia was shown to be able to correct the mutant Fah gene and rescue expression of the wild-type Fah protein in _ 1 out of 250 cells [43].

\section{Moving beyond Endogenous Cellular Repair}

For instance, the highly efficient DNA damage repair system in Deinococcus radiodurans [44]. May be exploited to enable efficient genome editing in mitotic as well as postmitotic cells. Furthermore, the majority of CRISPR-based technology development has focused on the signature Cas9 nuclease from type II. CRISPR systems. However, there remains a wide diversity of CRISPR types and functions. Cas RAMP module (Cmr) proteins identified in Pyrococcus furiosus and Sulfolobus solfataricus [45]. Constitute an RNAtargeting CRISPR immune system, forming a complex guided by small CRISPR RNAs that target and cleave complementary RNA instead of DNA.

However, numerous challenges still lie ahead. Most importantly, successful clinical translation will depend on appropriate and efficacious delivery systems to target specific disease tissues. To achieve high levels of therapeutic efficacy and simultaneously address a broad spectrum of genetic disorders, homologous recombination efficiency will need to be significantly improved. Although permanent genome modification has advantages over monoclonal antibody or siRNA treatments, which require repeated administration of the therapeutic molecule, the long-term implications remain unclear. As researchers further develop and test Cas9 toward clinical translation, it will be paramount to thoroughly characterize the safety as well as physiological effects of Cas9 using a variety of preclinical models [2].

\section{Endogenous Fluorescence Tagging by CRISPR}

The fluorescent protein GFP is inserted 50 to the stop codon of a gene of interest by including a gRNA mediated cut at a suit able PAM site (close to the stop codon). 50 and 30 homology arms of different length up to $1500 \mathrm{bp}$ have been used for HDR. For high efficiency, the donor fragment is flanked by additional CRISPR cleavage sites to provide a linear fragment for HDR. MMEJ is used with high efficiency to gene edit target cells. Short (micro) homology domains are used to knock-in GFP. In HDR, ssDNA strand invasion into the donor fragment occurs. In MMEJ nuclease resection causes single strands over the whole micro homology domain, which produces 'sticky ends' due to the presence of the same micro homology domains in the genome. More recently, NHEJ has been used for gene editing. Upon gRNA mediated cleavage a linear fragment is provided in the nucleus (e.g., by a donor vector, which includes the GFP flanked by CRISPR cleavages sites), which is then inserted in some cells by NHEJ repair [46].

\section{NHEJ-Driven CRISPR Knock-ins}

NHEJ is highly efficient and more likely than HDR, especially in eukaryotic cells; however, specificity of integration is hampered by INDELs (insertions and deletions), which might cause frame shifts, particularly problematic for knock-ins of fluorescents proteins (fusion genes).The NHEJ repair system has been used to integrate desired sequences into the duck enteritis virus genome, and GFP was inserted in between UL26 and UL27 loci [47]. NHEJ was also used to integrate large $10-\mathrm{kb}$ donor plasmids with up to $30 \%$ efficiency in various human and murine cell lines [48] linear donor fragment for integration is provided to Cas9-cleaved cells causing integration by NHEJ. High efficiency was obtained by flanking the donor fragment with Cas9cleavage sites and cloning of this cassette into a vector backbone. By this approach, regular highly efficient transfection was applicable, and generation of the linear dsDNA fragment is produced close to the locus of editing in the nucleus [49]. Figure2 showing NHEJ and HDR repair system. 


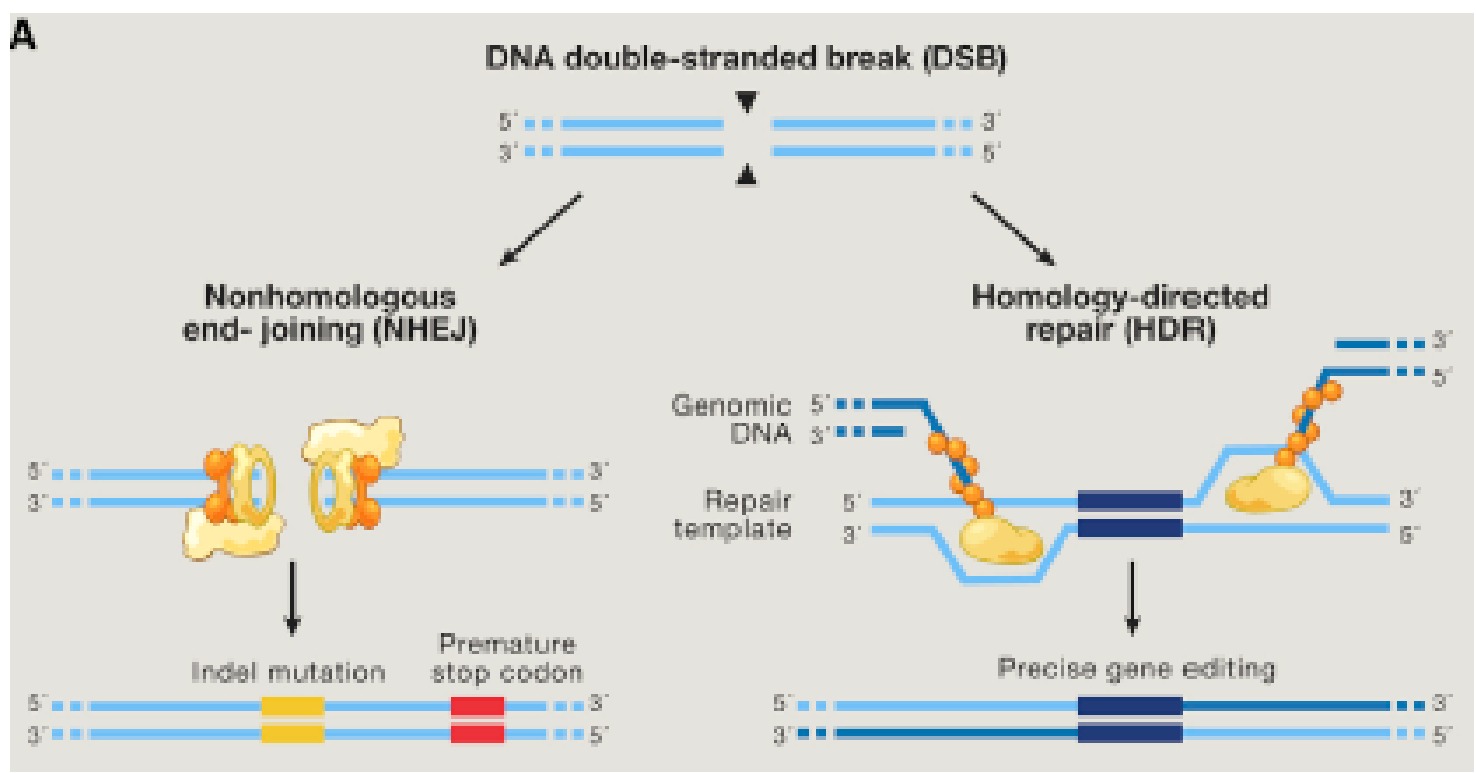

Fig-2: Figure showing NHEJ and HDR repair system

\section{Uniqueness of Fluorescent Protein Knock-ins}

Endogenous gene tagging using fluorescent proteins corresponds to a valuable tool to understand gene and protein function in cells or more complex model systems; for example, a labeled protein is detectable in live cells over long time periods, thus enabling a thorough study of its subcellular localization [46].

Fluorescent proteins like GFP have a size of $27 \mathrm{kDa}$ and thus, the corresponding donor needs to be flanked by large homology arms while using HDR. Besides helping to decipher important biological questions, use of fluorescent protein tagging also enables a fast generation of clonal cell lines due to the fact that these cells are easy to monitor using fluorescence microscopy. Although addition of suitable linkers, for example, GS linkers, can ensure proper folding and stability of the protein the large sized GFP tag to smaller protein might still influence their localization, interaction, stability, and dynamics. Stem cell knock-ins are still challenging, potentially due to some unique properties of the DNA repair machinery of those cells [50].

\section{CONCLUSION}

The latest development of Cas9-based genome engineering tools are also based on components from the microbial antiphage defense system. Rapid development of genome editing with the endonuclease systems has dramatically changed the biomedical research. The researches on nucleases for genome manipulation will revolutionize medical cares for many complex genetic diseases in the future.

\section{REFERENCES}

1. Gupta, D., Bhattacharjee, O., Mandal, D., Sen, M. K., Dey, D., Dasgupta, A.,... \& Chattopadhyay, D.
(2019). CRISPR-Cas9 system: A new-fangled dawn in gene editing. Life sciences, 116636.

2. Hsu, P. D., Lander, E. S., \& Zhang, F. (2014). Development and applications of CRISPR-Cas9 for genome engineering. Cell, 157(6), 1262 1278.[20] Gilbert, L. A., Larson, M. H., Morsut, L., Liu, Z., Brar, G. A., Torres, S. E., ... \& Lim, W. A. (2013). CRISPR-mediated modular RNAguided regulation of transcription in eukaryotes. Cell, 154(2), 442-451.

3. Jinek, M., Chylinski, K., Fonfara, I., Hauer, M., Doudna, J. A., \& Charpentier, E. (2012). A programmable dual-RNA-guided DNA endonuclease in adaptive bacterial immunity. science, 337(6096), 816-821.

4. Barrangou, R., Fremaux, C., Deveau, H., Richards, M., Boyaval, P., Moineau, S., ... \& Horvath, P. (2007). CRISPR provides acquired resistance against viruses in prokaryotes. Science, 315(5819), 1709-1712.

5. Marraffini, L. A. (2015). CRISPR-Cas immunity in prokaryotes. Nature, 526(7571), 55-61.

6. Mali, P., Yang, L., Esvelt, K. M., Aach, J., Guell, M., DiCarlo, J. E., ... \& Church, G. M. (2013). RNA-guided human genome engineering via Cas9. Science, 339(6121), 823-826.

7. Perez-Pinera, P., Kocak, D. D., Vockley, C. M., Adler, A. F., Kabadi, A. M., Polstein, L. R., ... \& Guilak, F. (2013). RNA-guided gene activation by CRISPR-Cas9-based transcription factors. Nature methods, 10(10), 973.

8. Chakraborty, S., Ji, H., Kabadi, A. M., Gersbach, C. A., Christoforou, N., \& Leong, K. W. (2014). A CRISPR/Cas9-based system for reprogramming cell lineage specification. Stem cell reports, 3(6), 940-947.

9. Hwang, W. Y., Fu, Y., Reyon, D., Maeder, M. L., Tsai, S. Q., Sander, J. D., ... \& Joung, J. K. (2013). Efficient genome editing in zebrafish using a 
CRISPR-Cas system. Nature biotechnology, 31(3), 227-229.

10. Puchta, H., \& Fauser, F. (2013). Gene targeting in plants: 25 years later. International Journal of Developmental Biology, 57(6-7-8), 629-637.

11. Puchta, H., \& Fauser, F. (2013). Gene targeting in plants: 25 years later. International Journal of Developmental Biology, 57(6-7-8), 629-637.

12. Barrangou, R., \& Horvath, P. (2012). CRISPR: new horizons in phage resistance and strain identification. Annual review of food science and technology, 3, 143-162.

13. Jiang, W., Zhou, H., Bi, H., Fromm, M., Yang, B., \& Weeks, D. P. (2013). Demonstration of CRISPR/Cas9/sgRNA-mediated targeted gene modification in Arabidopsis, tobacco, sorghum and rice. Nucleic acids research,41(20):e188e188.

14. Jiang, W., Brueggeman, A. J., Horken, K. M., Plucinak, T. M., \& Weeks, D. P. (2014). Successful transient expression of Cas9 and single guide RNA genes in Chlamydomonas reinhardtii. Eukaryotic cell, 13(11), 1465-1469.

15. Kao, P. H., \& Ng, I. S. (2017). CRISPRi mediated phosphoenolpyruvate carboxylase regulation to enhance the production of lipid in Chlamydomonas reinhardtii. Bioresource technology, 245, 1527-1537.

16. Deng, X., Cai, J., Li, Y., \& Fei, X. (2014). Expression and knockdown of the PEPC1 gene affect carbon flux in the biosynthesis of triacylglycerols by the green alga Chlamydomonas reinhardtii. Biotechnology letters, 36(11), 21992208.

17. Tanwar, A., Sharma, S., \& Kumar, S. (2018). Targeted genome editing in algae using CRISPR/Cas9. Indian Journal of Plant Physiology, 23(4), 653-669.

18. Spitz, F., \& Furlong, E. E. (2012). Transcription factors: from enhancer binding to developmental control. Nature reviews genetics, 13(9), 613-626.

19. Sera, T. (2009). Zinc-finger-based artificial transcription factors and their applications. Advanced drug delivery reviews, 61(7-8), 513-526.

20. Chen, B., Gilbert, L. A., Cimini, B. A., Schnitzbauer, J., Zhang, W., Li, G. W., ... \& Huang, B. (2013). Dynamic imaging of genomic loci in living human cells by an optimized CRISPR/Cas system. Cell, 155(7), 1479-1491.

21. Cheng, A. W., Wang, H., Yang, H., Shi, L., Katz, Y., Theunissen, T. W., ... \& Jaenisch, R. (2013). Multiplexed activation of endogenous genes by CRISPR-on, an RNA-guided transcriptional activator system. Cell research,23(10), 11631171.

22. Giménez, C. A., Ielpi, M., Mutto, A., Grosembacher, L., Argibay, P., \& Pereyra-Bonnet, F. (2016). CRISPR-on system for the activation of the endogenous human INS gene. Gene therapy, 23(6), 543-547.

23. Hu, J., Lei, Y., Wong, W. K., Liu, S., Lee, K. C., He, X., ... \& Peng, X. (2014). Direct activation of human and mouse Oct4 genes using engineered TALE and Cas9 transcription factors. Nucleic acids research, 42(7), 4375-4390.

24. Polstein, L. R., \& Gersbach, C. A. (2015). A lightinducible CRISPR-Cas9 system for control of endogenous gene activation. Nature chemical biology, 11(3), 198-200.

25. Yang, X. (2015). Applications of CRISPR-Cas9 mediated genome engineering. Military Medical Research, 2(1), 11.

26. Niu, Y., Shen, B., Cui, Y., Chen, Y., Wang, J., Wang, L., ... \& Xiang, A. P. (2014). Generation of gene-modified cynomolgus monkey via Cas9/RNA-mediated gene targeting in one-cell embryos. Cell, 156(4), 836-843.

27. Hwang, W. Y., Fu, Y., Reyon, D., Maeder, M. L., Tsai, S. Q., Sander, J. D., ... \& Joung, J. K. (2013). Efficient genome editing in zebrafish using a CRISPR-Cas system. Nature biotechnology, 31(3):227-229.

28. Shalem, O., Sanjana, N. E., Hartenian, E., Shi, X., Scott, D. A., Mikkelsen, T. S., ... \& Zhang, F. (2014). Genome-scale CRISPR-Cas9 knockout screening in human cells. Science, 343(6166), 8487.

29. Qi, L. S., Larson, M. H., Gilbert, L. A., Doudna, J. A., Weissman, J. S., Arkin, A. P., \& Lim, W. A. (2013). Repurposing CRISPR as an RNA-guided platform for sequence-specific control of gene expression. Cell, 152(5):1173-1183.

30. Konermann, S., Brigham, M. D., Trevino, A. E., Joung, J., Abudayyeh, O. O., Barcena, C., ... \& Nureki, O. (2015). Genome-scale transcriptional activation by an engineered CRISPR-Cas9 complex. Nature, 517(7536), 583.

31. Vickers, N. J. (2017). Animal Communication: When I'm Calling You, Will You Answer Too?. Current Biology, 27(14), R713-R715.

32. Zhou, H., Liu, J., Zhou, C., Gao, N., Rao, Z., Li, H., ... \& Sun, Y. (2018). In vivo simultaneous transcriptional activation of multiple genes in the brain using CRISPR-dCas9-activator transgenic mice. Nature neuroscience, 21(3), 440-446.

33. Doerr, A. (2015). Structural biology: Cryo-EM strikes gold. Nature methods, 12(2):102.

34. Han, K., Holder Jr, J. L., Schaaf, C. P., Lu, H., Chen, H., Kang, H., ... \& Yu, P. (2013). SHANK3 overexpression causes manic-like behaviour with unique pharmacogenetic properties. Nature, 503(7474), 72-77.

35. Cong, L., Ran, F. A., Cox, D., Lin, S., Barretto, R., Habib, N., ... \& Zhang, F. (2013). Multiplex genome engineering using CRISPR/Cas systems. Science, 339(6121), 819-823.

36. Bogerd, H. P., Kornepati, A. V., Marshall, J. B., Kennedy, E. M., \& Cullen, B. R. (2015). Specific 
induction of endogenous viral restriction factors using CRISPR/Cas-derived transcriptional activators. Proceedings of the National Academy of Sciences, 112(52), E7249-E7256.

37. Benitez, A. A., Panis, M., Xue, J., Varble, A., Shim, J. V., Frick, A. L., ... \& Sachs, D. (2015). In vivo RNAi screening identifies MDA5 as a significant contributor to the cellular defense against influenza A virus. Cell reports, 11(11), 1714-1726.

38. Doehle, B. P., Schäfer, A., \& Cullen, B. R. (2005). Human APOBEC3B is a potent inhibitor of HIV-1 infectivity and is resistant to HIV-1 Vif. Virology, 339(2), 281-288.

39. Wang, Y., Ding, Y., \& Li, J. (2017). CRISPRCas9-mediated gene editing in mouse spermatogonial stem cells. In RNAi and Small Regulatory RNAs in Stem Cells (pp. 293-305). Humana Press, New York, NY.

40. Long, C., McAnally, J. R., Shelton, J. M., Mireault, A. A., Bassel-Duby, R., \& Olson, E. N. (2014). Prevention of muscular dystrophy in mice by CRISPR/Cas9-mediated editing of germline DNA. Science, 345(6201), 1184-1188.

41. Ebina, H., Misawa, N., Kanemura, Y., \& Koyanagi, Y. (2013). Harnessing the CRISPR/Cas9 system to disrupt latent HIV-1 provirus. Scientific reports, 3, 2510.

42. Hu, W., Kaminski, R., Yang, F., Zhang, Y., Cosentino, L., Li, F., ... \& Mo, X. (2014). RNAdirected gene editing specifically eradicates latent and prevents new HIV-1 infection. Proceedings of the National Academy of Sciences, 111(31), 11461-11466.

43. Yin, H., Xue, W., Chen, S., Bogorad, R. L., Benedetti, E., Grompe, M., ... \& Anderson, D. G. (2014). Genome editing with Cas9 in adult mice corrects a disease mutation and phenotype. Nature biotechnology, 32(6), 551-553.

44. Yin, H., Xue, W., Chen, S., Bogorad, R. L., Benedetti, E., Grompe, M., ... \& Anderson, D. G. (2014). Genome editing with Cas9 in adult mice corrects a disease mutation and phenotype. Nature biotechnology, 32(6), 551-553.

45. Hale, C. R., Majumdar, S., Elmore, J., Pfister, N., Compton, M., Olson, S., ... \& Terns, M. P. (2012). Essential features and rational design of CRISPR RNAs that function with the Cas RAMP module complex to cleave RNAs. Molecular cell, 45(3), 292-302.

46. Bukhari, H., \& Müller, T. (2019). Endogenous Fluorescence Tagging by CRISPR. Trends in cell biology.

47. Chang, P., Yao, Y., Tang, N., Sadeyen, J. R., Sealy, J., Clements, A., ... \& Iqbal, M. (2018). The application of NHEJ-CRISPR/Cas9 and Cre-Lox system in the generation of bivalent duck enteritis virus vaccine against avian influenza virus. Viruses, 10(2), 81.

48. Tálas, A., Kulcsár, P. I., Weinhardt, N., Borsy, A., Tóth, E., Szebényi, K., ... \& Gordos, B. (2017). A convenient method to pre-screen candidate guide RNAs for CRISPR/Cas9 gene editing by NHEJmediated integration of a 'self-cleaving'GFPexpression plasmid. DNA Research,24(6), 609621.

49. Suzuki, K., Tsunekawa, Y., Hernandez-Benitez, R., Wu, J., Zhu, J., Kim, E. J., ... \& Kurita, M. (2016). In vivo genome editing via CRISPR/Cas9 mediated homology-independent targeted integration. Nature, 540(7631), 144-149.

50. Chen, X., Zaro, J. L., \& Shen, W. C. (2013). Fusion protein linkers: property, design and functionality. Advanced drug delivery reviews, 65(10), 1357-1369. 